\title{
Aplicación del tiempo de tromboplastina parcial activado (TTPA) con tiempo de incubación modificado en el diagnóstico del anticoagulante lúpico $(\mathrm{AL})$
}

\author{
Patricia Fardella B ${ }^{1}$, Pradip K Rustagi ${ }^{2}$ \\ Clinical application of the activated \\ partial thromboplastin time (APTT) with \\ modified incubation time for the \\ diagnosis of lupus anticoagulant (LA)
}

Background: Although several tests are used to screen for the presence of LA, none detects all its types. The shortening of APTT observed when the pre-incubation period is prolonged, proved to be a sensitive test for the presence of LA. Material and methods: We determined the APTT, performed with a 4 or 15 min preincubation period (APTTs and APTT15 respectively), in 22 healthy subjects, 3 commercial positive controls for LA, 16 patients with a previous diagnosis of LA and 54 patients with recurrent fetal loss and/or infertility. Evidence of LA was established by a positive Staclot-LA test. Results: APTTs and APTT15 were $31.5 \pm 4.7$ and $28.4 \pm 4.5$ seconds respectively in samples from 22 normal subjects. The figures in samples with LA, were $71.5 \pm 20.3 \mathrm{~s}$ and $58.6 \pm 18 \mathrm{~s}$ respectively. The difference between the two APTTs performed on an individual sample was defined as the APTT 4-15 and was 2.6 \pm 2.0 in normal subjects $2.5 \pm 2.8$ in 13 patients anticoagulated with warfarin, $-10.0 \pm 6.5$ in 13 patients receiving heparin, and $13.2 \pm 4.9$ in 15 patients with LA. The test values for LA patients were significantly higher than those for normal subjects ( $\mathrm{p}<0.0001$ ). For values over 5 , the APTT 4-15 had 93.3\% sensitivity and 100\% specificity. In one patient with recurrent fetal loss or infertility, who was LA positive, the APTT 4-15 was positive with a value of 14 . Conclusions: This modified TTPA is easy to perform, and provides a reasonably discriminatory value for the presence of LA. Therefore, we recommend the TTPA 4-15 to screen for LA (Rev Méd Chile 2004; 132: 407-12).

(Key Words: Antiphospholipid syndrome; Lupus coagulation inhibitor; pregnancy, highrisk)

\footnotetext{
Recibido el 15 de abril, 2003. Aceptado en versión corregida el 23 de diciembre, 2003. ${ }^{1}$ Servicio de Hematología, Hospital Clínico Universidad de Chile. Santiago, Chile. ${ }^{2}$ Laboratorio de Coagulación, Hospital de la Universidad de Alabama en Birmingham (UAB), Estados Unidos de Norteamérica.
}

Correspondencia a: Dra. Patricia Fardella B. Hospital Clínico de la Universidad de Chile. Santos Dumont 999, Santiago, Chile. Fax: (562) 6788248. E mail: pfardella123@msn.com 
$\mathrm{E}^{1}$ anticoagulante lúpico (AL) corresponde a inmunoglobulinas que interfieren in vitro con las pruebas de coagulación dependientes de fosfolípidos ${ }^{1}$.

Se utilizan muchas pruebas para detectar AL, de ellas, el TTPA es la más usada como método de screening, pero su sensibilidad varía según el reactivo ${ }^{2}$. Las pruebas confirmatorias: veneno de víbora de Russell diluido confirmatorio, neutralización plaquetaria, neutralización con antifosfolípidos hexagonales II (Staclot-LA), también son múltiples, complejas y ninguna detecta todos los $\mathrm{AL}^{3}$. En 1994, Robert y cols ${ }^{4}$ describieron una nueva prueba que modificaba los tiempos de incubación del TTPA, que era positiva para AL cuando la diferencia entre el TTPA con 1 y $10 \mathrm{~min}$ de incubación (TTPA 1-10) era $>11 \mathrm{~s}$, sensible y específica para AL si se asociaba a los estudios de mezcla y presentaba como falsos (+) los déficits de factores de contacto (Prekalikreína y F XII). Esta prueba fue repetida por Cloherty y cols, quienes observaron una menor sensibilidad para la detección de $\mathrm{AL}^{5}$.

La prevalencia de AL en la población general de embarazadas es alrededor de $0,2 \%$, en pacientes con más de 2 pérdidas fetales es $7-8 \%{ }^{7}$, y más alta si se asocia a lupus eritematoso sistémico $^{8}$. Los mecanismos involucrados en estas pérdidas fetales y si ellas son embrionarias o predominantemente fetales aún no han sido completamente aclarados ${ }^{9-}$ 11. En pacientes con infertilidad, se ha descrito una asociación con la presencia de anticuerpos antifosfolípidos, tanto del tipo anticardiolipina (ACA) como $\mathrm{AL}$, pero hasta el momento esta relación no ha sido claramente establecida ${ }^{12-14}$.

El propósito de este estudio fue evaluar esta prueba de menor costo y complejidad en pacientes con diagnóstico de LA, en normales, en terapia anticoagulante oral y con heparina no fraccionada. Además, aplicar esta prueba en una población obstétrica con mayor probabilidad de presentar AL por sus antecedentes clínicos, como una forma de establecer la utilidad de ella.

\section{Pacientes y MÉTodos}

Se realizó la prueba TTPA 4-15 en 22 controles sanos, obtenidos del laboratorio de coagulación, los cuales presentaban TTPA, tiempo de protrombina y de trombina normales, en 3 controles positivos de AL estándar (Cryo Lupus and G King Lupus CCT), en 16 pacientes con diagnóstico previo de AL, estudiado en relación al antecedente de enfermedad autoinmune (12 pacientes) e hipertensión pulmonar primaria en 4; todos ellos tenían TTPA prolongado (>50 s previo al estudio), no corregía con la mezcla plasma normal 1:1, la prueba confirmatoria con Staclot-LA fase II fue $>8$ s (positivo), el tiempo de trombina normal y se descartaron otros inhibidores específicos de la coagulación. Se estudiaron 13 pacientes que se encontraban en terapia con warfarina y 13 pacientes en terapia anticoagulante con heparina no fraccionada.

Se estudiaron además, en forma retrospectiva 54 pacientes del Servicio de Ginecología y Obstetricia del Hospital de la Universidad de Alabama en Birmingham con diagnóstico de pérdidas fetales recurrentes o infertilidad. Las características de las pacientes se muestran en la Tabla 1.

Las muestras de sangre fueron obtenidas por punción venosa en tubos con citrato de sodio, se centrifugaron por $5 \mathrm{~min}$ a $29.000 / \mathrm{rpm}$ y el plasma fue filtrado con filtros de $0,45 \mu$ a $0,22 \mu$. Las muestras se congelaron a $-70^{\circ} \mathrm{C}$ hasta ser procesadas.

El plasma normal fue obtenido de Cryo Check ${ }^{\mathrm{TC}}$ Citrated Human Plasma. Las pruebas de coagulación se realizaron en un equipo STA System (Diagnostica Stago) y el Staclot LA fase (II) en un coagulómetro mecánico de punto final (ST4, Diagnostica Stago).

Para el TTPA estándar (TTPAs) se usó como reactivo STA-PTT-LT que contiene cefalina y sílica como activador (Diagnostica Stago), plasma pobre en plaquetas y el tiempo de preincubación fue de $4 \mathrm{~min}$; el TTPA15 fue realizado con $15 \mathrm{~min}$ de preincubación con los mismos reactivos. Los anticuerpos anticardiolipinas IgG, IgM e IgA se realizaron por método de ELISA.

La prueba TTPA 4-15 se definió como la diferencia entre TTPAs y TTPA15 y se realizó en todos los pacientes previamente descritos.

Los resultados se muestran como promedios \pm desviación estándar. Para la comparación entre grupos se utilizó la prueba t de Student para muestras no pareadas. Se realizó curva ROC para determinar el punto de corte de la prueba. 


\section{RESUltados}

Los resultados de TTPA con tiempo de incubación modificado en plasma normal y en AL fueron los siguientes (promedio \pm DS): en plasma normal TTPAs fue $31,5 \pm 4,7 \mathrm{~s}$ y el TTPA15 $28,4 \pm 4,5 \mathrm{~s}$. El TTPA15 se acorta en forma significativa respecto al estándar ( $p<0,01)$ en los pacientes normales. En las 18 muestras positivas para $\mathrm{AL}$, que incluye control positivo y pacientes el TTPAs fue $71,5 \pm 20,3$ seg y el TTPA15 de 58,6 \pm 18 s. De los 16 pacientes con AL previo, se excluyó 1 que presentó un TTPA normal, por no cumplir con el primer criterio diagnóstico para AL, aunque previamente presentaba TTPA prolongado. En los 15 pacientes restantes el TTPAs fue $74 \pm 21 \mathrm{~s}$, el TTPA15 60,8 $\pm 18,8$ s y el TTPA $4-15$ 13,2 $\pm 4,9$. En la Tabla 2 se muestran los resultados individuales de TTPAs, TTPA15 y TTPA 4-15 en controles, pacientes con AL y controles AL positivos.

Los resultados de la prueba TTPA 4-15 para todos los pacientes estudiados se muestran en la Tabla 3, donde se observa que tanto los pacientes con AL como los controles $(+)$ presentan valores prolongados en relación a los normales $(p<0,0001)$. Los pacientes en terapia con warfari- na muestran valores similares a los controles normales, sólo 1 paciente de este grupo presentó un valor elevado para la prueba TTPA 4-15, en él se descartó la presencia de AL por una prueba confirmatoria (Staclot LA) que fue negativa. En los pacientes en terapia con heparina no fraccionada se produjo una prolongación del TTPA15 determinando valores negativos para la prueba, este grupo también presenta diferencias significativas con los controles normales pero en el sentido opuesto a los pacientes con AL ( $p$ $<0,0001$ ).

La prueba TTPA 4-15 $\geq 5$ s fue considerada positiva para AL con una sensibilidad de 93,3\%, especificidad de $100 \%$ y un intervalo de confianza 95\% de 0,853-0,996. El área bajo la curva ROC fue 0,97 y el error estándar 0,032.

De las 54 pacientes obstétricas evaluadas, 3 presentaban ACA (+) en bajos títulos y una de ellas presentaba tanto ACA como AL positivos. En todas ellas se aplicó la prueba TTPA 4-15, en las 53 pacientes sin $\mathrm{AL}$, la prueba tuvo un valor de 2,9 \pm 2 (Promedio \pm DS), valor similar a los controles normales; sin embargo en la paciente con $\mathrm{AL}$ (+) la prueba TTPA 4-15 tuvo un valor de 14, con un TTPAs de 62 y TTPA15 de 48.

Tabla 1. C aracterísticas de las pacientes obstétricas

\begin{tabular}{|lcccc|}
\hline & $\begin{array}{c}\text { Grupo I } \\
(\mathrm{n}=30)\end{array}$ & $\begin{array}{c}\text { Grupo II } \\
(\mathrm{n}=14)\end{array}$ & $\begin{array}{c}\text { Grupo III } \\
(\mathrm{n}=10)\end{array}$ & $\begin{array}{c}\text { Total } \\
(\mathrm{n}=54)\end{array}$ \\
\hline Edad (X+DS) & $30,1 \pm 5,2$ & $31,9 \pm 5,3$ & $32,1 \pm 4,3$ & $31,1 \pm 5,1$ \\
Pérdidas fetales (X+DS) & $3,7 \pm 1,4$ & $2,1 \pm 0,7$ & $0,7 \pm 0,5$ & $2,4 \pm 1,6$ \\
Parto prematuro & 3 & 1 & 0 & 4 \\
RCIU* & 2 & 0 & 0 & 2 \\
Pre eclampsia & 1 & 0 & 0 & 1 \\
Cefalea & 1 & 1 & 3 & 3 \\
Trombosis & 1 & 0 & 2 & 2 \\
Accidentes vasculares & 1 & 0 & 4 & 10 \\
Endometriosis & 2 & 4 & 0 & 3 \\
Historia de AL (+)* & 1 & 1 & 0 & 5 \\
Historia de ACA (+)* & 2 & 3 & 0 & 3 \\
Historia de ANA (+)* & 2 & 0 & 4 & 5 \\
\hline
\end{tabular}

Grupo I: Pacientes con antecedentes de pérdidas fetales recurrentes. Grupo II: Pacientes con antecedentes de pérdidas fetales recurrentes e infertilidad. Grupo III: Pacientes con antecedentes de infertilidad. *RCIU: Retardo del crecimiento intrauterino. AL: anticoagulante lúpico. ACA: anticuerpos anticardiolipinas. ANA: anticuerpos antinucleares. 


\section{DisCUSIÓN}

El AL es un anticuerpo que prolonga los tiempos de coagulación por medio de unión a fosfolípidos utilizando cofactores como $\aleph_{2}$ glicoproteína-I ( $\Omega_{2}$ GP I) y protrombina. Sus manifestaciones clínicas son múltiples y entre las más frecuentes observa- mos las trombosis venosas y arteriales, abortos recurrentes ${ }^{15,16}$ y trombocitopenia ${ }^{17}$.

La búsqueda de pruebas de menor complejidad y costo para el diagnóstico de AL es relevante ya que ninguna los identifica a todos. Entre las más utilizadas tenemos:

Tabla 2. Valores de T T PAs, T T PA15 y T T PA 4-15 en controles, pacientes con anticoagulante lúpico (AL) positivo y controles positivos

\begin{tabular}{|c|c|c|c|c|c|c|c|c|}
\hline \multicolumn{3}{|c|}{$\begin{array}{c}\text { Controles } \\
n=22\end{array}$} & \multicolumn{3}{|c|}{$\begin{array}{c}\text { Pacientes AL (+) } \\
n=15\end{array}$} & \multicolumn{3}{|c|}{$\begin{array}{c}\text { Controles AL (+) } \\
n=3\end{array}$} \\
\hline $\begin{array}{l}\text { TTPAs } \\
\text { seg }\end{array}$ & $\begin{array}{c}\text { TTPA15 } \\
\text { seg }\end{array}$ & ТТРА 4-15 & $\begin{array}{l}\text { TTPAs } \\
\text { seg }\end{array}$ & $\begin{array}{l}\text { TTPA15 } \\
\text { seg }\end{array}$ & TTPA 4-15 & $\begin{array}{l}\text { TTPAs } \\
\text { seg }\end{array}$ & $\begin{array}{l}\text { TTPA15 } \\
\text { seg }\end{array}$ & ТTРА 4-15 \\
\hline 34 & 29 & 5 & 57 & 42 & 15 & 61 & 51 & 10 \\
\hline 32 & 28 & 4 & 45 & 42 & 3 & 61 & 51 & 10 \\
\hline 34 & 29 & 5 & 83 & 63 & 20 & 54 & 40 & 14 \\
\hline 34 & 30 & 4 & 55 & 45 & 10 & & & \\
\hline 36 & 33 & 3 & 130 & 115 & 15 & & & \\
\hline 32 & 27 & 5 & 69 & 56 & 13 & & & \\
\hline 37 & 32 & 5 & 75 & 56 & 19 & & & \\
\hline 36 & 31 & 5 & 59 & 50 & 9 & & & \\
\hline 36 & 33 & 3 & 67 & 58 & 9 & & & \\
\hline 35 & 32 & 3 & 100 & 81 & 19 & & & \\
\hline 39 & 42 & -3 & 75 & 56 & 19 & & & \\
\hline 26 & 24 & 2 & 101 & 84 & 17 & & & \\
\hline 28 & 26 & 2 & 58 & 45 & 13 & & & \\
\hline 26 & 25 & 1 & 74 & 63 & 11 & & & \\
\hline 25 & 25 & 0 & 63 & 57 & 6 & & & \\
\hline 26 & 21 & 4 & & & & & & \\
\hline 24 & 22 & 2 & & & & & & \\
\hline 36 & 33 & 3 & & & & & & \\
\hline 25 & 24 & 1 & & & & & & \\
\hline 29 & 27 & 2 & & & & & & \\
\hline 27 & 26 & 1 & & & & & & \\
\hline 36 & 26 & 0 & & & & & & \\
\hline X 31,5 & 28,4 & 2,6 & 74 & 60,8 & 13,2 & 58,6 & 47,3 & 11,3 \\
\hline DS 4,7 & 4,5 & 2 & 21,3 & 18,8 & 5 & 3,3 & 5,2 & 1,9 \\
\hline
\end{tabular}

TTPAs: TTPA realizado con 4 min de incubación. TTPA15: TTPA realizado con 15 min de incubación. TTPA 45: diferencia entre TTPAs y TTPA15.

Tabla 3. Resultados de la prueba T T PA 4-15

\begin{tabular}{|c|c|c|c|c|c|}
\hline & $\begin{array}{c}\text { Normales } \\
(n=22)\end{array}$ & $\begin{array}{l}\mathrm{AL}(+) \\
(\mathrm{n}=18)\end{array}$ & $\begin{array}{l}\text { Warfarina } \\
(\mathrm{n}=13)\end{array}$ & $\begin{array}{c}\text { Heparina } \\
(\mathrm{n}=13)\end{array}$ & $\begin{array}{l}\text { Pts Obstétricas } \\
(\mathrm{n}=53)\end{array}$ \\
\hline TTPA 4-15 (X土DS) & $2,6 \pm 2$ & $12,9 \pm 4,6$ & $2,5 \pm 2,8$ & $-9,6 \pm 7,1$ & $2,9 \pm 2$ \\
\hline Rango & $0-5$ & $3-20$ & $(-3)-9$ & $(-25)-(-1)$ & $(-2)-8$ \\
\hline
\end{tabular}


a) TTPA: es muy utilizada pero tiene el inconveniente de que los reactivos varían en su capacidad para detectar el $\mathrm{AL}$, los mejores para monitorear terapias con heparina no son buenos para detectar $\mathrm{AL}$; no es una buena prueba aun usando los reactivos más sensibles. b) Tiempo de coagulación con Kaolin: TTPA sin fosfolípido en el reactivo, lo que lo hace muy sensible para AL, mezcla proporciones variables de plasma del paciente y plasma normal. Su inconveniente es que requiere realizar múltiples pruebas de laboratorio tanto del paciente como del normal ${ }^{18}$. c) Veneno de víbora de Russell diluido (dRVVT): utiliza veneno de víbora que es activador del factor $\mathrm{X}$ y fosfolípidos en forma micelar, los que son diluidos para hacerlos más sensibles para AL Es una de las mejores pruebas para detectar $\mathrm{AL}$, especialmente en pacientes con TTPA normal; no es afectado por inhibidores de factor VIII, factor IX o factor XI, pero sí por heparina, warfarina e inhibidores de factor $\mathrm{V}^{19}$. d) Inhibición de tromboplastina tisular: el tiempo de protrombina se prolonga más en pacientes con LA que en normales al diluir la tromboplastina. Usa fosfolípidos unidos a proteína y tiene como falsos positivos inhibidores de factor VIII, factor IX y heparina. No es una buena prueba diagnóstica ${ }^{20}$.

La confirmación del AL detectado por las pruebas previamente mencionadas, requiere la utilización de fosfolípidos que deben acortar los tiempos. Entre ellos tenemos: a) Veneno de víbora de Russell diluido confirmatorio: el mismo principio que el anterior pero corrige con fosfolípidos, idealmente sin utilizar fosfolípidos plaquetarios en la corrección por la presencia de factor plaquetario 4 , pues pueden producir falsos positivos en presencia de heparina. El ideal es utilizar cefalina ${ }^{21}$. b) Test de neutralización plaquetaria: compara los valores de TTPA en presencia de plaquetas como fosfolípidos y solución salina, discrimina entre inhibidores específicos (especialmente factor VIII) y AL, la heparina produce falsos positivos ${ }^{22}$. c) Corrección con fosfolípidos hexagonales (Staclot LA): usa liposomas de fosfatidiletanolamina (PE), que neutralizan AL mejor que otros fosfolípidos ya que forman fases hexagonales. En esta prueba se comparan los TTPA adicionando PE y sin PE y usando un reactivo sensible para $A L$; en presencia de AL el TTPA con PE es más corto. La prueba es positiva si el valor de TTPA sin PE menos el valor de TTPA con PE es $\geq 8 \mathrm{~s}$. Esta prueba no se altera con inhibidores específicos ni con heparina en niveles $<1 \mathrm{U} / \mathrm{mL}^{23}$. d) TTPA con fosfolípido diluido: utiliza sílica como activador, fosfolípido de cerebro de bovino en diferentes diluciones y plasma normal para eliminar el efecto del déficit de factores. En AL los TTPA son 5 veces mayores que los normales; tiene como falsos positivos los inhibidores de factor VIII $>10 \mathrm{BU} / \mathrm{ml}^{24}$.

La prueba utilizada por nosotros, que posee una alta sensibilidad y especificidad para detectar $\mathrm{AL}$, se basa en el acortamiento del TTPK al aumentar el tiempo de incubación, el que es significativamente mayor en portadores de AL. La causa de este fenómeno no es clara pero se atribuye a la acción del AL en la fase de contacto de la coagulación, donde estaría involucrada la $\aleph_{2}$-GP-I. La $\aleph_{2}$-GP-I es una proteína plasmática que neutraliza las macromoléculas de carga negativa que entran a la circulación; también se sabe que inhibe la fase de contacto, por acción en el factor XII y factor XIIa a través de prekalikreí$\mathrm{na}^{25-27}$, lo que le confiere una actividad anticoagulante. Se ha observado que el complejo anti$\aleph_{2}$-GP-I/ $\S_{2}$-GP-I tiene una actividad semejante a AL y es capaz de aumentar la activación del factor XII cuando se encuentra en concentraciones elevadas $^{28}$ y éste puede ser uno de los mecanismos implicados en el mayor acortamiento del TTPA en pacientes con $\mathrm{AL}$.

El fenómeno que se observa en los pacientes tratados con heparina se debe a una acción inhibitonia sobre el factor XII, por lo que al aumentar el tiempo de incubación el TTPA se prolonga, determinando valores negativos para la prueba.

Cloherty y cols ${ }^{5}$ observaron que la sensibilidad de la prueba está relacionada con el tipo de activador utilizado en el TTPA y la mayor sensibilidad se obtiene cuando se utiliza sílica como activador.

La prueba TTPA4-15, demostró ser sensible y específica para detectar AL En la población obstétrica estudiada, sólo una paciente fue positiva para $\mathrm{AL}$, y este hallazgo fue confirmado por la nueva prueba. En base a estos resultados lo recomendamos como prueba de screening para AL teniendo en cuenta que cada laboratorio debe determinar los tiempos de incubación más adecuados según los reactivos y equipos utilizados. 


\section{REFERENCIAS}

1. Bick RL Antiphospholipid thrombosis syndromes. Hematol/Oncol Clinics of North America 2003; 17: 115-47.

2. Denis-Magdeiaine A, Fiahault A, Verdy E. Sensitivity of sixteen APTT reagents for the presence of lupus anticoagulants. Haemostasis 1995; 25: 98-105.

3. TRIPIETT DA, BARNA LK, Unger GA. A Hexagonal (II) Phase Phospholipid Neutralization Assay for Lupus Anticoagulant Identification. Thromb Haemost 1993; 70: 787-93.

4. RoberT A. Two Different Incubation Times for the Activated Partial Thromboplastin Time (APTT): a new criterion for diagnosis of lupus anticoagulants. Thromb Haemost 1994; 71: 220-4.

5. Cloherty T, Golden EA, LIND SE. Use of a modified Activated Partial Thromboplastin Time to detect Lupus Anticoagulants. Thromb Research 1996; 83: 137-42.

6. Pattison NS, Chamey LW, McKay EJ, Lggins GC, BUTLER WS. Antiphospholipid antibodies in pregnancy: prevalence and clinical associations. Br J Obstet Gynecol 1993; 100: 909-13.

7. Maclfan MA, Cumming GP, McCall F, Walker ID, WALKER JJ. The prevalence of lupus anticoagulant and anticardiolipin antibodies in women with history of first trimester miscamiages. Br J Obstet Gynecol 1994; 101: 103-6.

8. Lockwood CJ, JACOB HR. The immunobiology and obstetrical consequences of antiphospholipid antibodies. Obstet Gynecol Surv 1994; 49: 432-9.

9. Rai RS, ClifFord K, Cohen H, Regan L High prospective fetal loss rate in untreated pregnancies of women with recurrent miscarriage and antiphospholipid antibodies. Hum Reprod 1995; 10: 3301-4.

10. Festin MR, Limson GM, Mauro T. Autoimmune causes of recurrent pregnancy loss. Kobe J Med Sci 1997; 43: $143-57$.

11. LAKASING L, Poston L Adverse pregnancy outcome in the antiphospholipid syndrome: focus for future research. Lupus 1977; 6: 681-4.

12. GHAZEERI GS, KuTteH WH. Autoimmune factors in reproductive failure. Curr Opin Gynecol 2001; 13: 287-91.

13. BRANCH DW. Antiphospholipid antibodies and reproductive outcome: the current state of affairs. J Reprod Inmunol 1998; 38: 75-87.

14. Balasch J, Creus M, Fabregues F, Civico S, Reverter JC, Carmona F et aL Antiphospholipid antibodies and the outcome of pregnancy after the first in vitro fertilization and embryo transfer cycle. Hum Reprod 1998; 13: 1180-3.

15. TRIPLETT D. Lupus Anticoagulants/Antiphospholipid protein antibodies: the great imposters. Lupus 1996; 5: 431-5.

16. LevY RA. Clinical manifestations of the APL syndrome. Lupus 1996; 5: 393-7.

17. Gaw M, Finazzi G, Barbui T. Thrombocytopenia in the antiphospholipid syndrome: pathophysiology, clinical relevance and treatment. Ann Med Interne 1996; 147 (Suppl 1): 24-7.

18. EXNER T, RichaRD KA, KRONENBERg H. A sensitive test demonstrating lupus anticoagulant and its behavioural pattems. Br J Haematol 1978; 40: 143-51.

19. Thiagarajan P, Pengo V, Shapiro S. The use of the dilute Russell viper venom time for the diagnosis of lupus anticoagulant. Blood 1986; 68: 869-74.

20. Schieider MA, Nachman RL, JafFe EA, Coleman M. A clinical study of the lupus anticoagulant. Blood 1976; 48: 499-509.

21. Weinstein C, Muer M, Axtens R. Livido reticularis associated with increased titers of anticardiolipin antibodies in systemic lupus erythematosus. Arch Dermatol 1987; 123: 596-600.

22. Trippiet DA, Brandt JT, Kaczor D, SchaefFer J. Laboratory diagnosis of lupus inhibitor: A comparison of the tissue thromboplastin inhibition procedure with a new platelets neutralization procedure. Am J Clin Pathol 1983; 79: 678-82.

23. EXNER T. Diagnosis methodologies for circulating anticoagulants. Thromb and Haemost 1995; 74: 33844.

24. Alving BM, Baidwin PE, Richards RL, Jackson BJ. The dilute phospholipid APTT: A sensitive assay for verification of lupus anticoagulants. Thromb and Haemost 1985; 54: 709-12.

25. Schousboe I. Beta 2-Glycoprotein I: a plasma inhibitor of the contact activation of the intrinsic blood coagulation pathway. Blood 1985; 66: 1086-91.

26. Henry ML, Everson B, Ratnoff OD. Inhibition of the activation of Hageman factor (factor XII) by $\beta_{2}$ glycoprotein I. J Lab Clin Med 1988; 11: 519-23.

27. SchousBoe I. Inositolphospholipid accelerated activation of prekallikrein by activated factor XII and its inhibition by $\aleph_{2}$-glycoprotein I. Eur J Biochem 1988; 176: 629-36.

28. Schousboe I, Rasmussen M. Synchronized inhibition of the phospholipid mediated autoactivation of Factor XII in plasma by $\aleph_{2}$-Glycoprotein I and Anti- $\aleph_{2}$-Glycoprotein I. Tromb Haemost 1995; 73: 798-804. 